\title{
Financiarisation des marchés de matières premières et conséquences
}

\section{(2) OpenEdition \\ Journals}

Édition électronique

URL : http://journals.openedition.org/economierurale/4073

DOI : $10.4000 /$ economierurale.4073

ISSN : 2105-2581

Éditeur

Société Française d'Économie Rurale (SFER)

Édition imprimée

Date de publication : 15 octobre 2013

Pagination : XX

ISSN : 0013-0559

\section{Référence électronique}

«Financiarisation des marchés de matières premières et conséquences », Économie rurale [En ligne], 337 | septembre-octobre 2013, mis en ligne le 15 octobre 2015, consulté le 19 avril 2019. URL : http:// journals.openedition.org/economierurale/4073; DOI : 10.4000/economierurale.4073 


\section{DÉBAT}

\section{Financiarisation des marchés de matières premières et conséquences}

La rubrique " Débat » d'Économie rurale s'appuie désormais sur des interventions dans le cadre des Séminaires de politiques agricoles organisés par la Société française d'économie rurale. Sur un sujet d'actualité, deux points de vue courts ${ }^{1}$ sont demandés aux intéressés. Cette fois-ci, il s'agit de développer la question de la financiarisation des marchés de matières premières, et notamment les incidences sur les prix agricoles et alimentaires. Trois auteurs, Nicolas Maystre, David Bicchetti (Conférence des Nations Unies pour le Commerce et le Développement - CNUCED -) et Bernard Valluis (Association Nationale de la Meunerie Française - ANMF -) se sont prêtés au jeu, sur la base de leur intervention dans le SPA de septembre 2012.

\section{Points de vue de David Bicchetti et Nicolas Maystre, économistes à la CNUCED}

Ce texte s'inspire de plusieurs publications publiées par la Conférence des Nations Unies pour le Commerce et le Développement indiquées en référence ${ }^{2}$. Néanmoins, les opinions exprimées dans cet article sont celles des auteurs et ne doivent pas être considérées comme les points de vue officiels de la CNUCED ou de ses États membres.

es prix des produits de base ont connu des fluctuations considérables au cours de la dernière décennie. Leur envolée entre 2002 et 2008 a été la plus forte depuis plusieurs dizaines d'années de par son ampleur, sa durée et sa portée. La contraction des prix survenue ensuite après le déclenchement de la crise mondiale actuelle au milieu de l'année 2008 a été particulièrement forte et a touché un nombre important de produits. Depuis le milieu de l'année 2009 et surtout depuis l'été 2010, les prix mondiaux des

1. De moins de 9000 signes chacun.

2. CNUCED (2011a). Rapport sur le commerce et le développement, 2011. New York et Genève, $\mathrm{Pu}-$ blication des Nations Unies, UNCTAD/TDR/2011, numéro de vente : F.11II.D.3. CNUCED (2012). Don't blame the physical markets: financialization is the root cause of oil and commodity price volatility, Policy Brief, $\mathrm{n}^{\circ} 25$, septembre. produits de base ${ }^{3}$ sont néanmoins repartis à la hausse, mais ils se sont quelque peu stabilisés par la suite.

Pour certains, cette récente évolution des prix des produits de base s'explique par les profondes transformations des relations fondamentales entre l'offre et la demande, notamment la forte croissance des économies émergentes couplées (i) à une urbanisation rapide et à des changements de régimes alimentaires de leurs classes moyennes ; (ii) à des tentatives de réduire l'utilisation des énergies fossiles et au

3. Dans le langage courant, on parle souvent de «matière première ». Néanmoins, cette terminologie ne reflète pas le fait que certains de ces produits sont le fruit d'opérations de transformations de haute technologie, donc éloignés du concept originel de matière première. C'est pourquoi on utilise davantage le terme de «produit de base » dans la littérature. 
recours aux biocarburants ; (iii) à la diminution de la productivité de certains produits agricoles liée à des chocs d'offre en lien ou non avec le réchauffement climatique.

Néanmoins, les analyses valident de plus en plus l'idée que ces fluctuations s'expliquent aussi par la présence de plus en plus intense des investisseurs financiers dans le négoce des produits de base pour des motivations purement financières, souvent qualifiées de « financiarisation du négoce des produits de base ».

Depuis le début du millénaire, les investissements dans les matières premières (ou leurs dérivés) sont devenus un élément dans des stratégies plus vastes d'allocation de portefeuille des investisseurs. Cela s'est traduit par une augmentation significative des actifs liés aux produits de base qui sont passés de moins de 10 milliards de dollars vers la fin du siècle dernier à un niveau record de 458 milliards de dollars en avril 2011 (Barclays, 2012) ${ }^{4}$. Ainsi, les volumes des produits dérivés en cours (open contracts) négociés en bourse sur les marchés des matières premières sont aujourd'hui 20 à 30 fois supérieurs à la production physique (Silvennoinen et Thorp, 2010) ${ }^{5}$.

Bien que la participation des acteurs financiers aux marchés des produits de base soit un élément normal des marchés, toute la question consiste à savoir de quelle ampleur sont ces flux financiers et dans quelle mesure ils déconnectent les prix par rapport aux fondamentaux et/ou contribuent à l'instabilité des prix. En théorie, la participation de ces acteurs pourrait être bénéfique du point de vue économique si elle contribue à rendre les marchés plus liquides et à mieux prendre en compte les

4. Barclays (2012). The Commodity Investor (divers numéros).

5. Silvennoinen A., Thorp S. (2010). Financialization, Crisis and Commodity Correlation Dynamics, Research Paper $n^{\circ}$ 267, Quantitative Finance Research Centre, University of Technology Sydney. besoins en matière de couverture des utilisateurs commerciaux et à réduire les coûts correspondants. Néanmoins, les avantages ainsi procurés peuvent aussi être anéantis par les comportements grégaires de ces acteurs suivant souvent des stratégies d'investissements qui répondent aux mêmes signaux. Du fait de cette financiarisation, les marchés des produits de base obéissent moins à la logique d'un marché normal de marchandises qu'à celle des marchés financiers.

\section{Mouvements corrélés sur les marchés des actions et des produits de base}

Il est difficile de déterminer dans quelle mesure le niveau et l'instabilité des prix des produits de base sont liés aux investissements financiers, faute de transparence et de données suffisamment détaillées. Il existe néanmoins des éléments qui étayent l'idée que les investisseurs financiers influencent la dynamique des prix, du moins dans le court terme. Il peut notamment être fait référence aux fortes corrélations récentes existant entre les mouvements des prix des produits de base et les évolutions sur les marchés des actions, ces derniers étant par nature extrêmement financiarisés.

Historiquement, les prix des produits de base et des actions évoluaient habituellement en sens opposé (Gorton et Rouwenhorst, 2006) ${ }^{6}$. Néanmoins, dès 2009, la CNUCED a commencé à signaler de plus fortes corrélations positives entre les prix des matières premières et ceux de plusieurs autres actifs financiers (CNUCED, 2009, 2011b) ${ }^{7}$.

6. Gorton G., Rouwenhorst K.G. (2006). Facts and Fantasies about Commodity Futures, Financial Analysts Journal, ${ }^{\circ}$ 62, p. 47-68.

7. CNUCED (2009). The Global Economic Crisis: Systemic Failures and Multilateral Remedies. Publication des Nations Unies, numéro de vente : E.09.II.D.4, New York et Genève, CNUCED (2011b). Price Formation in Financialized Commodity Markets: The Role of Information. Publication des Nations Unies, UNCTAD/GDS/2011/1, New York et Genève. 
Depuis, ces corrélations se sont renforcées. Dans un récent document de travail de la CNUCED, Bicchetti et Maystre (2012) ${ }^{8}$ ont analysé les co-mouvements entre les rendements sur plusieurs produits de base et sur le marché des actions aux États-Unis durant la période 1997-2011 à l'aide des données ventilées par échelon de cotation (données tick). Les données montrent l'apparition des co-mouvements positifs entre les rendements des contrats à terme du pétrole ou d'autres produits agricoles avec le marché des actions des États-Unis, y compris sur des intervalles temporels extrêmement courts. Dans le cas du pétrole, Bicchetti et Maystre (2012) ont été jusqu'à considérer des intervalles d'une seconde.

De toute évidence, ces mouvements simultanés à très court terme des prix des matières premières ne peuvent être justifiés par des variations de l'offre et de la demande propres à chacun des marchés des différents produits de base. Par ailleurs, les fondamentaux - à savoir, les principaux déterminants de l'offre et la demande sur un marché - entre les produits de base et le marché des actions des États-Unis sont en principe distincts. Dès lors, on ne s'attendrait pas à observer des corrélations positives à des fréquences si élevées et durant une si longue période, qui plus est sur une telle variété de produits de base. En effet, on s'attendrait à des comportements distincts en raison notamment des saisonnalités, de l'utilisation industrielle et physique, et plus généralement des dynamiques propres à chacun de ces produits de base. Étrangement, ces différences n'apparaissent pas réellement dans les observations. À l'inverse, les marchés boursiers et des matières premières partagent une caractéristique essentielle commune : la forte présence

8. Bicchetti D., Maystre N. (2012). The synchronized and long-lasting structural change on commodity markets: Evidence from high-frequency data, UNCTAD Discussion Papers, n 208 (UNCTAD/OSG/DP/2012/2). d'investisseurs financiers. Cela met en relief une autre conséquence préoccupante et jusqu'à présent sous-estimée de la financiarisation : sa capacité de porter préjudice à l'économie réelle en envoyant les mauvais signaux pour la gestion macroéconomique. En effet, les prix en vigueur sur un marché sont censés refléter, du moins en théorie, toute l'information disponible quant aux fondamentaux liés à ce produit. À partir du moment où des investisseurs financiers interviennent sur les marchés des dérivés des produits de base pour des motifs autres que ceux ayant trait à l'échange du sousjacent, les signaux émanant de ces marchés peuvent aller à l'encontre de l'évolution des fondamentaux.

Un exemple récent éclaire ce changement. Les graphiques 1 et 2 illustrent l'évolution des prix de l'Euro Stoxx $600^{\circ}$, le prix du pétrole brut WTI - l'indice de référence aux États-Unis - et l'indice des matières premières de Standard \& Poor et Goldman Sachs (SPGSCI) de février à septembre 2002 et 2012, respectivement.

En comparant l'évolution de ces prix au cours de 2012 avec celle d'il y a dix ans, on s'aperçoit que la financiarisation des marchés de produits révèle un changement radical. Malgré certaines similitudes entre 2002 et 2012 en termes de chocs réels - insécurité au Moyen-Orient, lendemain d'un krach boursier et faibles récoltes pour certaines céréales -, l'évolution des trois indices ne pourrait être plus divergente. Il y a dix ans chaque marché avait sa propre dynamique, mais en 2012, ils se déplacent en tandem.

En particulier, en 2012, les prix du SPGSCI et du WTI suivent de près les événements qui se déroulent dans la zone euro. Ces similitudes sont d'autant plus

9. L'Euro Stoxx 600 est un indice boursier au niveau européen. Au même titre que le CAC 40 pour la France, cet indice est basé sur le prix des actions de 600 sociétés au sein de 18 pays européens. 
Graphique 1. Évolution de l'Euro Stoxx 600, du pétrole brut WTI et de l'indice des matières premières de Standard \& Poor et Goldman Sachs (SPGSCI) - 31 janvier au 30 septembre 2002

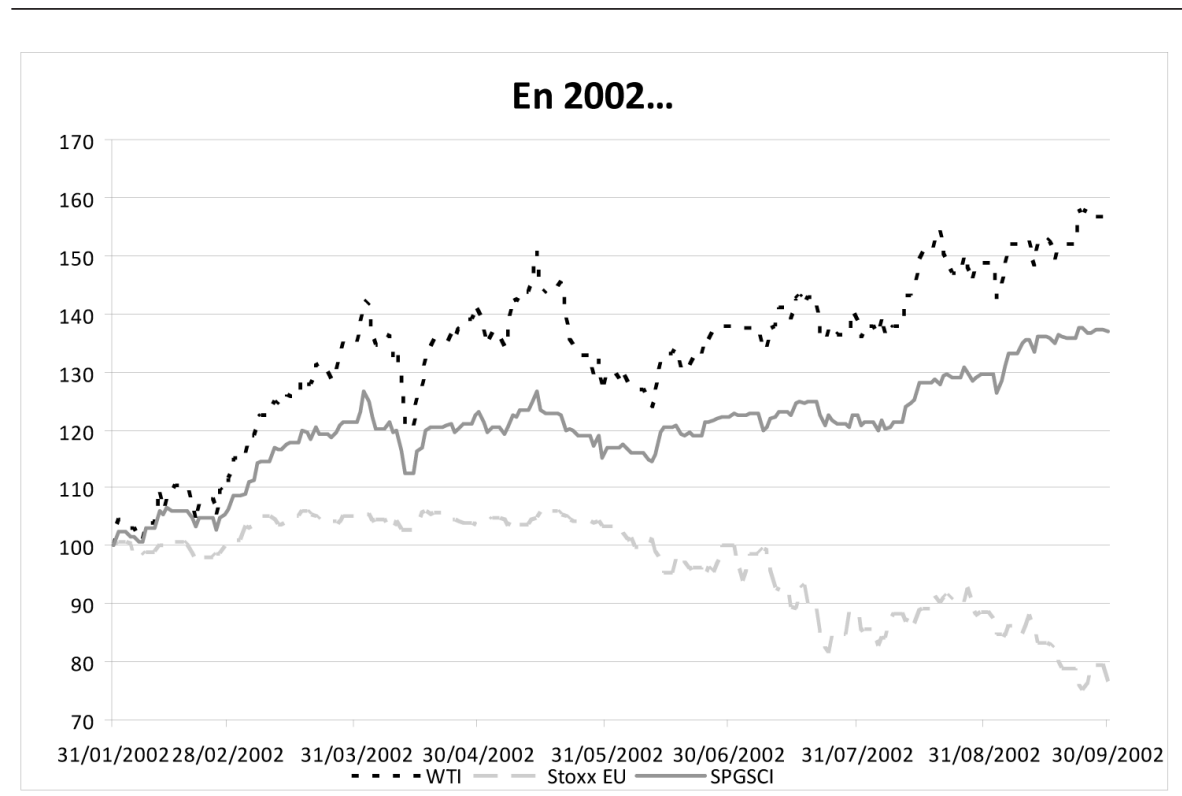

Note : Indices de prix (prix au 31/01/2002 = 100)

Source : calculs du secrétariat de la CNUCED basés sur la base de données de Bloomberg.

frappantes que, contractuellement, la livraison du sous-jacent aux contrats à terme sur le pétrole brut WTI reste confinée au Midwest des États-Unis, ce qui restreint grandement les possibilités d'arbitrage avec le pétrole utilisé en Europe. Dès lors, il est difficile de comprendre pourquoi la consommation de pétrole qui est livrée à Cushing dans l'Oklahoma est si dépendante de la conjoncture en Europe.

Une autre illustration de l'influence des investisseurs financiers sur les marchés des produits de base se trouve dans le redressement sur les marchés pétroliers qui s'est opéré suite à l'accord de recapitalisation des banques dans la zone euro à la fin de juin 2012. À cette époque, le prix du Brent a augmenté de $7 \%$ en une seule journée et celui du WTI de $9 \%$ - une augmentation, bien visible sur le graphique 2, qui n'était pas liée à des changements fondamentaux de l'offre et de la demande dans les mois à venir.

\section{Des mesures contre l'instabilité des prix des produits de base}

Des mesures d'urgence à court terme s'imposent pour prévenir ou atténuer les effets néfastes de la financiarisation des prix des produits de base. Dans le même temps, il faut réfléchir aux moyens d'améliorer le fonctionnement des marchés de produits dérivés adossés aux produits de base pour permettre à ces mécanismes de mieux remplir leur rôle en envoyant des signaux de prix valables aux producteurs et aux consommateurs de produits de base, ou au moins pour les empêcher d'envoyer les mauvais signaux.

Compte tenu du rôle vital de l'information dans l'évolution des prix des produits de base, quatre mesures devraient permettre, prises ensemble, d'améliorer le fonctionnement de ces marchés, surtout pour les denrées alimentaires et pour l'énergie. 
Graphique 2. Évolution de l'Euro Stoxx 600, du pétrole brut WTI et de l'indice des matières premières de Standard \& Poor et Goldman Sachs (SPGSCI) - 31 janvier au 30 septembre 2012

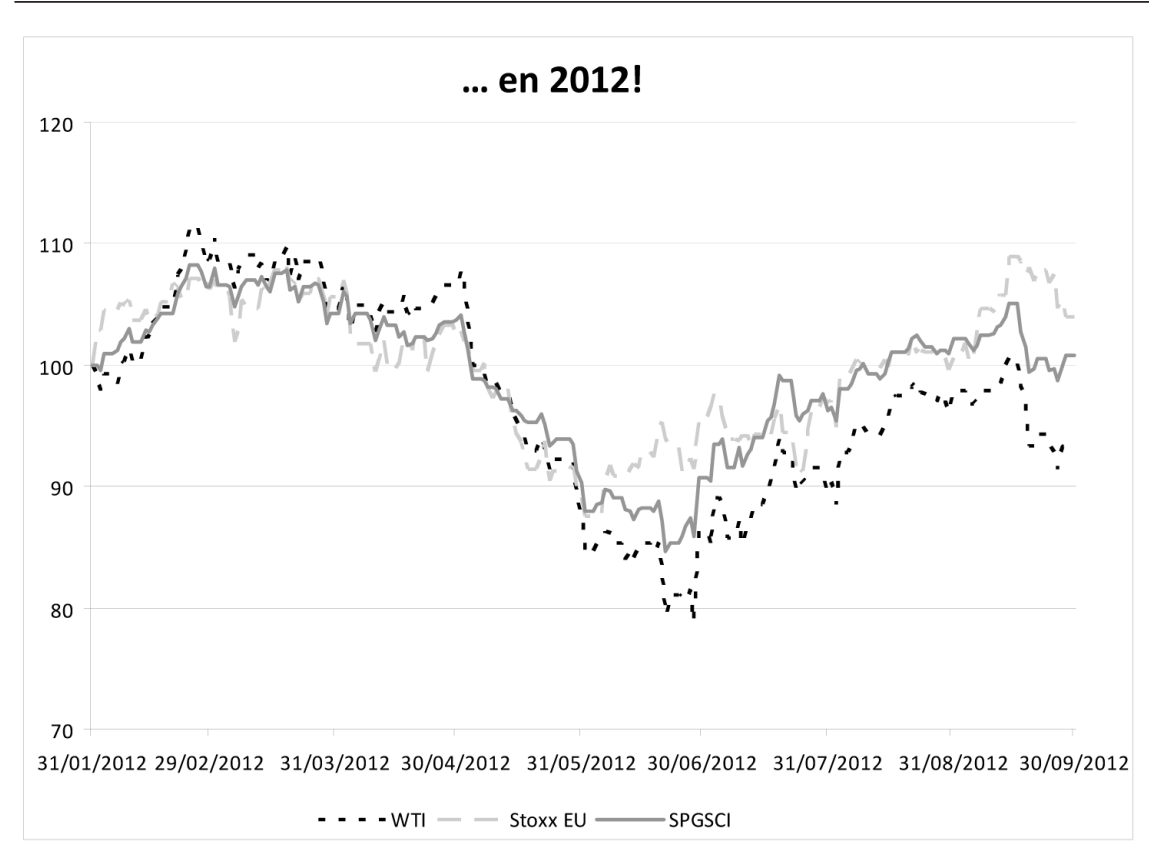

Note : Indices de prix (prix au 31/01/2012 = 100)

Source : calculs du secrétariat de la CNUCED basés sur la base de données de Bloomberg.

- Premièrement, il faudrait accroître la transparence des marchés physiques en fournissant des informations plus fiables et plus actuelles sur les produits de base, concernant par exemple les capacités disponibles et les stocks détenus au niveau mondial pour le pétrole, et les superficies plantées, les récoltes attendues, les stocks et les prévisions de demande à court terme pour les produits agricoles. Cette mesure permettrait aux acteurs commerciaux de mieux apprécier les rapports entre l'offre et la demande des produits de base à l'heure actuelle et dans l'avenir.

- Deuxièmement, il faudrait améliorer les flux d'information sur les marchés de produits dérivés adossés aux produits de base ainsi que l'accès à ces flux, notamment concernant les prises de position des différents opérateurs sur les marchés de ces produits, ce qui permettrait également de gagner en transparence. En particulier, des mesures pour assujettir les opérations sur les bourses en Europe à des obligations en matière d'information comparables à celles en vigueur aux États-Unis devraient considérablement améliorer la transparence des opérations et décourager le « nomadisme réglementaire », à savoir la migration vers des places boursières moins régulées.

- Troisièmement, il conviendrait de renforcer la réglementation des acteurs financiers, par exemple en plafonnant le volume des positions, pour limiter l'impact des investisseurs financiers sur les marchés des produits de base. Et il faudrait interdire aussi les transactions pour compte propre aux institutions financières qui effectuent des opérations de couverture au bénéfice de leurs clients, pour cause de conflit d'intérêts. Cela impliquera de trouver le 
juste milieu entre une réglementation trop restrictive, qui empêcherait les bourses de produits de remplir leurs fonctions de transfert des risques, et une réglementation trop laxiste, qui les empêcherait aussi de s'acquitter de leurs fonctions de base.

- Quatrièmement, les autorités de surveillance des marchés pourraient être chargées d'intervenir directement, mais ponctuellement, sur les marchés des produits de base en achetant ou en vendant des contrats de produits dérivés pour éviter un effondrement des prix ou pour dégonfler des bulles de prix. Cette intervention pourrait être considérée comme une solution de dernier recours pour lutter contre la formation de bulles spéculatives s'il n'était pas adopté de mesures de réforme pour assurer une plus grande transparence des marchés et leur réglementation plus rigoureuse, ou bien au cas où ces mesures se révéleraient inefficaces. Si le mécanisme de déclenchement des interventions pouvait être essentiellement fondé sur des règles, et prévisible par conséquent, l'intervention proprement dite impliquerait nécessairement une certaine part de jugement et de pouvoir discrétionnaire pour les autorités de surveillance. Or il a parfois été émis des doutes quant à la capacité des autorités de marché ou des institutions publiques de comprendre et de suivre le marché. Ces doutes sont sans fondement car il n'y a aucune raison que ces organismes n'appréhendent pas le problème tout aussi bien que les autres acteurs du marché. Sur les marchés qui se prêtent à des comportements grégaires, chacun a accès aux mêmes informations. En outre, contrairement aux autres acteurs du marché, l'autorité chargée d'intervenir n'aurait aucune raison de s'engager dans une quelconque forme de comportement grégaire. Elle pourrait au contraire interrompre les chaînes d'information qui nourrissent de tels comportements en annonçant à un moment donné qu'elle considère que les prix ne reflètent absolument plus les fondamentaux.

\section{Le point de vue de Bernard Valluis, président délégué de l'ANMF}

Pour les investisseurs financiers, les produits dérivés ayant pour sous-jacents des matières premières sont désormais devenus des actifs banalisés auxquels sont appliquées les mêmes stratégies d'allocation des capitaux qu'aux autres classes d'actifs financiers. Les évolutions technologiques dans le trading, la recherche d'actifs pour limiter les risques de dépréciation liés à l'inflation et la dérégulation des marchés financiers à partir des années 1980 comptent parmi les facteurs à l' origine de cette évolution.

Le développement de ces nouvelles finalités dans l'usage de la finance sur matières premières s'est accompagné de la mise au point d'instruments de marché de plus en plus sophistiqués. Ainsi, à côté des places de marchés organisés, les produits dérivés traités de gré à gré (Over the counter ou OTC) ont connu un essor considérable : les volumes de ces transactions dépassent aujourd'hui largement ceux des marchés organisés.

Par rapport à ces derniers, les marchés de gré à gré présentent en effet l'avantage de coûts de transaction réduits, et de la confidentialité des opérateurs, mais également l'inconvénient d'exposer les cocontractants aux risques de défaillance de l'une des parties. Avec l'éclatement de différentes bulles spéculatives, et en particulier celle des subprimes, on a pu observer à quel point ces risques de défaut génèrent à leur tour des risques systémiques pour l'ensemble des marchés financiers, mais aussi et surtout économiques.

Le développement des produits dérivés a été par ailleurs, au cours de la première décennie du $\mathrm{XXI}^{\mathrm{e}}$ siècle, largement favorisé par leur mode de prise en compte dans l'établissement des bilans comptables des institutions financières, tel que prévu par les premières normes du Comité de Bâle. 
Ce mode de prise en compte, en sous-estimant le risque systémique induit par le manque de transparence et de contrepartie de ces produits, a fortement dégradé, on l'a constaté depuis, la résilience des institutions bancaires, et, par là même, tout le tissu financier et économique mondial.

Plus récemment, les développements du trading algorithmique et du trading haute fréquence ont eux aussi largement contribué aux changements observés dans la dynamique de la formation des prix sur les marchés dérivés de produits agricoles.

Dans la même période, le commerce des matières premières en général a fortement bénéficié du développement des économies des pays émergents, et les fondamentaux des marchés des matières premières agricoles ont connu de profondes modifications. Ainsi, leur cadre politicoréglementaire a largement évolué, tout particulièrement en Europe, avec le retrait des politiques d'intervention et de régulation des marchés, le découplage des mesures de soutien aux revenus et l'abaissement effectif des protections douanières.

Dans ces conditions, les marchés mondiaux des grandes cultures, céréales et oléagineux se sont élargis et largement globalisés, tant du point de vue des marchés physiques que des marchés financiers, tandis qu'ont pratiquement disparu tous les mécanismes d'intervention publique sur les marchés dans les principaux pays exportateurs.

Au-delà du constat que les matières premières en général, et les matières premières agricoles destinées à l'alimentation humaine en particulier, sont devenues une classe d'actifs banalisés, le thème des conséquences de cette financiarisation a ouvert un large débat pour les économistes, en amont des décisions politiques de réforme des marchés financiers.

\section{La financiarisation comme objet de recherches économiques}

Les travaux des économistes néoclassiques sont sous-tendus par l'hypothèse du fondement vertueux du marché, dont l'autorégulation permettrait la détermination d'un juste prix. Mais peut-on vraiment se satisfaire de l'hypothèse d'équilibre qui voudrait que le prix égalise le coût de production marginal à chaque instant, au vu de la volatilité des prix extrêmes observée depuis 2007 ? Il serait bienvenu que les économistes rediscutent des hypothèses de travail certes commodes mais qui les empêchent de comprendre la réalité de la formation des prix. L'hypothèse de l'efficacité des marchés formulée par Eugène Fama va dans ce sens, comme l'image souvent répétée que la «spéculation financière » n'aurait pas d'effet significatif sur les mécanismes de prix, qui ne dépendraient que des fondamentaux de chaque produit : «la spéculation ne serait que l'écume à la crête de la vague » pour reprendre l'expression chère au spécialiste des matières premières Philippe Chalmin.

Sans aborder directement la question de la financiarisation, les économistes des principales organisations internationales questionnées par le G20 agricole ont traité de la volatilité des marchés des matières premières. Les fondements analytiques de la volatilité structurelle des marchés agricoles théorisés par Gregory King ou Mordecai Ezekiel notamment sont soudain réapparus dans les rapports d'expertise alors que cette réalité semblait avoir été niée.

Les divergences relatives à la définition technique de la volatilité, les données disponibles et les modèles de traitement n'ont pas permis de dégager un consensus sur le constat d'un accroissement de la volatilité et l'établissement de relations de causalité avec les différentes facettes du processus de financiarisation. Aussi les débats 
académiques se poursuivent-ils sur cette question, mais force est de constater que le niveau de compréhension du fonctionnement des marchés et de la formation des prix reste très faible.

C'est donc tout le mérite des travaux de Nicolas Maystre et David Bicchetti, comme ceux de Jean-Jacques et Steve Ohana, de mettre en évidence, d'une part la forte corrélation entre les mouvements des produits de base et les évolutions sur les marchés des actions, d'autre part le rôle des fonds indiciels, dont les stratégies d'investissement font abstraction des fondamentaux des commodities qu'ils traitent.

C'est pourquoi on peut conclure que les marchés des matières premières sont aujourd'hui décorrélés, au moins partiellement, de leurs fondamentaux, que les marchés des produits dérivés correspondants ne sont plus en mesure de remplir normalement leur fonction d'arbitrage des transactions physiques et de révélateur du prix de transaction et que les signaux erronés qui s'en dégagent desservent l'économie réelle au plan macroéconomique.

En matière de méthodologie, il faut admettre que l'absence totale de transparence des marchés physiques comme des marchés des produits dérivés traités de gré à gré (OTC) et les données très partielles sur les marchés organisés de futures et d'options, ne permettent pas de disposer de toutes les données nécessaires pour la construction de modèles robustes au-delà des constats empiriques. Par ailleurs, sur la base du peu de données disponibles, la mise en correspondance des stratégies d'investisseurs et les phases de marchés correspondantes sont difficiles à mettre en évidence.

Parmi toutes les implications à venir des réformes financières en cours, l'amélioration de la transparence des marchés (pre trade et post trade) devrait contribuer à fournir les données nécessaires aux recherches économiques sur le sujet.

\section{La financiarisation comme objet de régulation}

Si les États-Unis, forts de leur longue expérience de régulation, disposent d'un niveau de transparence qui sera sensiblement amélioré par la mise en œuvre du Dodd-Franck Act, on est loin de disposer de données comparables en Europe. Par ailleurs, les transactions OTC multiplient les risques de contrepartie, et sont par là même source d'un risque systémique que la communauté internationale s'est résolu à ne plus tolérer. Or la faiblesse de la réglementation européenne en matière de prévention et de répression des abus et manipulations de marché est devenue d'autant plus criante et source de risque que ces marchés se sont développés.

L'amélioration de la transparence, la réduction des risques systémiques et la lutte contre les abus de marché ont ainsi fait partie des objectifs du G20, relayés par les travaux de l'Organisation internationale des commissions de valeur (OICV). Une profonde réforme des marchés OTC a ainsi été amorcée, prévoyant que ces contrats soient déclarés à un référentiel central, et que, à terme, ils soient majoritairement échangés sur des plates-formes de négociation et fassent l'objet d'une compensation.

L'Union européenne, dans le cadre des engagements pris au G20, a donc entamé une large refonte de sa réglementation sur les marchés financiers, par la régulation des infrastructures de marché (règlement UE 648-2012, dit EMIR) et des instruments financiers (règlement et directive MIF, en cours de négociation). Ce nouveau cadre réglementaire prévoit donc la migration de la majorité des OTC sur des plates-formes de négociation et leur compensation financière. Les marchés régulés, faisant déjà l'objet d'une compensation, devraient voir leur transparence accrue, grâce au suivi des positions, et leur fonctionnement sécurisé par l'établissement de limites d'emprise et/ou de position et la refonte de 
la réglementation sur les abus de marchés (directive et règlement MAD/MAR).

\section{La financiarisation comme révélateur des dysfonctionnements des marchés physiques}

Les progrès réalisés en matière de régulation financière mettent toutefois en évidence l'opacité qui subsiste sur la majorité des transactions physiques, et les risques que fait peser, en retour, cette opacité des marchés physiques sur les marchés financiers.

La financiarisation des matières premières agricoles suscite donc des interrogations sur les marchés sous-jacents, et doit nous conduire à remettre en cause les pratiques existantes sur les marchés physiques, et à leur appliquer les objectifs du G20. Ainsi, au vu de la financiarisation des marchés des céréales, la méconnaissance de l'état des stocks physiques représente une importante asymétrie d'information, et ouvre la porte aux bulles spéculatives voire aux manipulations. De plus, la mise en place du suivi des positions et des limites d'emprises financières doit, pour être efficace, trouver son pendant sur les marchés physiques. L'Union européenne a ainsi confirmé la légitimité des autorités de régulation financières dans le suivi des marchés sous-jacents, posant par là même la question de la mise en place de régulateurs sectoriels. En l'espèce, le modèle au niveau européen semble être constitué entre les régulateurs financiers et les régulateurs de l'énergie et surtout de l'électricité. Bien qu'érodés par les vents de la dérégulation, des instruments de connaissance et de suivi des marchés agricoles persistent, d'autres seront vraisemblablement à reconstruire, le tout devra être réorganisé via des régulateurs sectoriels agricoles, nationaux dans un premier temps, afin que les régulateurs financiers disposent des moyens nécessaires pour mettre en œuvre les prérogatives élargies que ce cadre de régulation renouvelé leur apporte. Il ne s'agit donc plus d'opposer régulation et marché : les marchés ont besoin de règles pour fonctionner. 